\title{
A Study of an Efficient ZigBee Address Assignment Scheme for Home Area Networks of Smart Grid
}

\author{
Dongmin Choi ${ }^{\dagger}$, llyong Chung ${ }^{+\dagger}$
}

\begin{abstract}
In Home Area Network (HAN) of the smart grid, Power Line Communication (PLC) technology and ZigBee communication technology can be used in the communication among the Advanced Metering Infrastructure (AMI) devices. However, according to performance evaluation results of the PLC technology, we find that using the PLC technology is unsuitable for the remote meter reading service. It is worth noting that some parts of the PLC are converted to the ZigBee communication technology in Jeju, Korea. Compared with PLC, ZigBee has no restriction of the place, where the equipments can be freely set up, due to the advantage of radio communication. However, number of usable devices will impact the network performance which is depended on the address assignment. In addition, due to the restriction of transmission range among devices, it is difficult to apply the ZigBee address assignment method to the practical circumstance. In this paper, we examine the previous ZigBee address assignment schemes and the corresponding routing algorithms, and propose a novel address assignment scheme compared with the existing methods, the performance of the proposed one is improved. In particular, evaluation results show that the proposed scheme reduces the average number of hop count, the transfer time and the processing time.
\end{abstract}

Key words: zigbee address, home network, smart grid

\section{INTRODUCTION}

Smart grid is the next-generation power grid where the power supplier and consumer exchanges the information with bidirectional on a real-time basis and optimizes the energy efficiency [1]. When we consider the countryside where people are living in sparse area, Power Line Communication (PLC) technology has a problem to support fre-

※ Corresponding Author: Ilyong Chung, Address: Dept. of Computer Engineering, Chosun University, 375 Seosuk-dong, Dong-gu, Gwangju 501-759, South Korea, TEL : +82-62-230-7712, FAX : +82-62-230-7754, E-mail : iyc@chosun.ac.kr

Receipt date: Dec. 20, 2012, Revision date : Feb. 28, 2013 Approval date: Mar. 26, 2013

${ }^{+}$Department of Computer Science, Chosun University (E-mail: cdm1225@gmail.com)

${ }^{+\dagger}$ Department of Computer Science, Chosun University ※ This work was supported by the research fund from Chosun University, 2012. An earlier version of this paper [14] was presented at the conference of Korean institute of smart media, Jeju University, April 19-20, 2012. quent multiplex signal of Advanced Metering Infrastructure (AMI) devices because of line speed limitation and line quality limitation. Thus, for maintain the link quality additional devices are required. In the case of ZigBee communication technology[2], has no restriction of the place, where the equipments can be freely set up and communicate each other within wireless communication coverage without support of additional $\mathrm{de}^{-}$ vices, due to the advantage of radio communication. Thus, many researchers proposed the applications [3-7] based on the basic ZigBee communication scheme. However, the number of usable devices, will impact the network performance which is depended on the address assignment [8-11]. In addition, due to the restriction of transmission range among devices, it is difficult to apply the ZigBee address assignment method to the practical circumstance.

The rest of this paper is organized as follows. 
Section 2 introduces some address assignment schemes based on ZigBee tree network. Section 3 explains our scheme in detail, and Section 4 evaluates the performance of the proposed scheme in terms of average number of hop count, transfer time, and processing time. Finally, we conclude the paper in Section 5.

\section{RELATED WORK}

\subsection{Distribute Address Assignment \& Tree Routing Algorithm}

The devices of ZigBee network are assigned their own network addresses by the distributed address assignment method (BAAM). Suppose that, the maximum number of child nodes is $C_{m}$, the maximum number of child routers is $R_{m}$, and the depth of the network is $L_{m}$. We may compute the children's address pool for each node according to the algorithm. Note that $\operatorname{Cskip}(d)$ is used to derive the starting address of its children's address pool. Cskip for the coordinator or a router in depth $d$ is defined as:

$\operatorname{Cskip}(d)=\left\{\begin{array}{cc}1+C_{m}\left(L_{m}-d-1\right) & , \text { if } R_{m}=1 \\ \frac{1+C_{m}-R_{m}-C_{m} \cdot R_{m}^{L_{m}-d-1}}{1-R_{m}} & \text {, otherwise }\end{array}\right.$

In addition, the parent node with depth $\mathrm{d}$ assigns 16bit address to the nodes who want to establish the connection with the parent node by equation (2) below.

$$
A_{n}=A_{\text {parent }}+\operatorname{Cskip}(d) \times m+1
$$

Where $A_{n}$ denotes the address assigned to the new node, $A_{\text {parent }}$ denotes the address of the parent node and $m$ denotes the number of routers of the parent node.

As we know, tree routing is a simple scheme in which data can be delivered to the parent node or child node. When a node receives data, it checks the destination information. If the destination information does not match, the node checks its child nodes addresses in order to match the destination information until meet the end point by equation (3).

$$
A<A_{\text {dest }}<A+\operatorname{Cskip}(d-1)
$$

Where $A$ denotes address of node itself and $A_{\text {dest }}$ denotes destination address. If $A_{d e s t}$ satisfies above condition, the packet is delivered to the next address calculated by equation (4), where $A_{r}$ denotes the address of the node where the packet will be delivered.

$$
A_{r=} A+1+\left\lfloor\frac{A_{\text {dest }}-(A+1)}{\operatorname{Cskip}(d)}\right\rfloor \times \operatorname{Cskip}(d)
$$

\subsection{Orphan node \& networks expansion limitation problem}

Fig. 1 shows an example of the orphan node problem in ZigBee distribute address assignment.

Node $N$ tries to join the network as a child node of node 7 or 10 . However, node 7 cannot assign the address since the number of child node has become 5 already. Note that the maximum number of child node is 5 . In addition, Node 10 cannot has a child node either, since the maximum depth of the tree topology is 2 . Therefore, node $N$ becomes orphan node.

In this case, when the connection of the node is concentrated on the partial domain of the tree, due to the restriction of the topology, even if the address field remains, the orphan node is generated. It is easily concluded that the maximum number of node participating in the network is 65,536 in the ZigBee network since the address uses 16 bits. In addition, if this network is expanded to the maximum length by using distribute address assignment scheme, the maximum depth becomes 15 .

We assume that the maximum communication distance of the node is $30 \mathrm{~m}$. Hence, the limitation of connectable distance is $450 \mathrm{~m}$. This limitation problem makes the application difficult. Therefore, this scheme is difficult to apply to the smart grid network environment. 


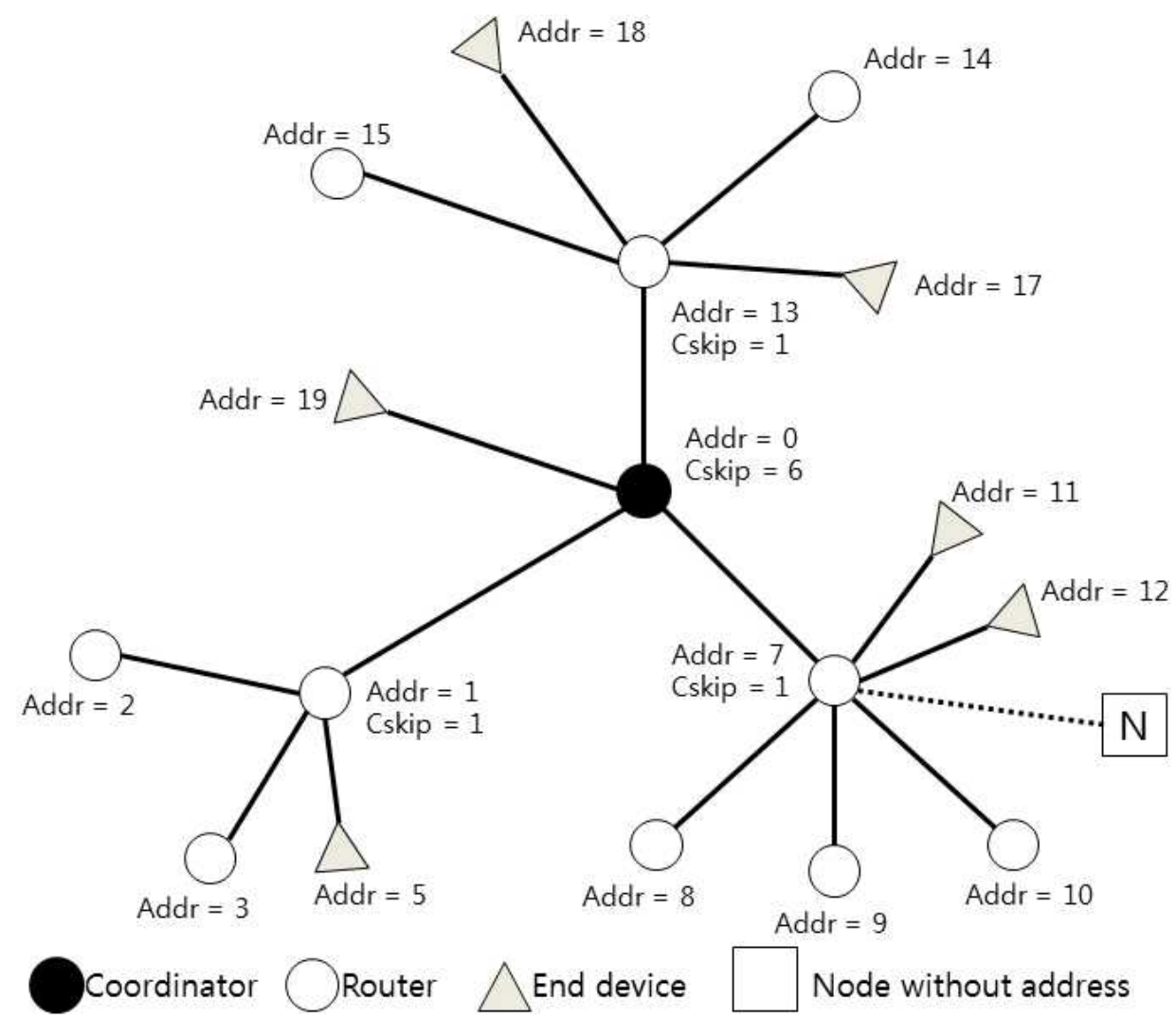

Fig. 1. Orphan problem $\left(C_{m}=5, R_{m}=3, L_{m}=2\right)$

2.3 Coordinate based address assignment method

In order to resolve the problem of the limitation of the connectable distance of the distribute address assignment, coordinate based address assignment method (CBAAM) was proposed in [12,

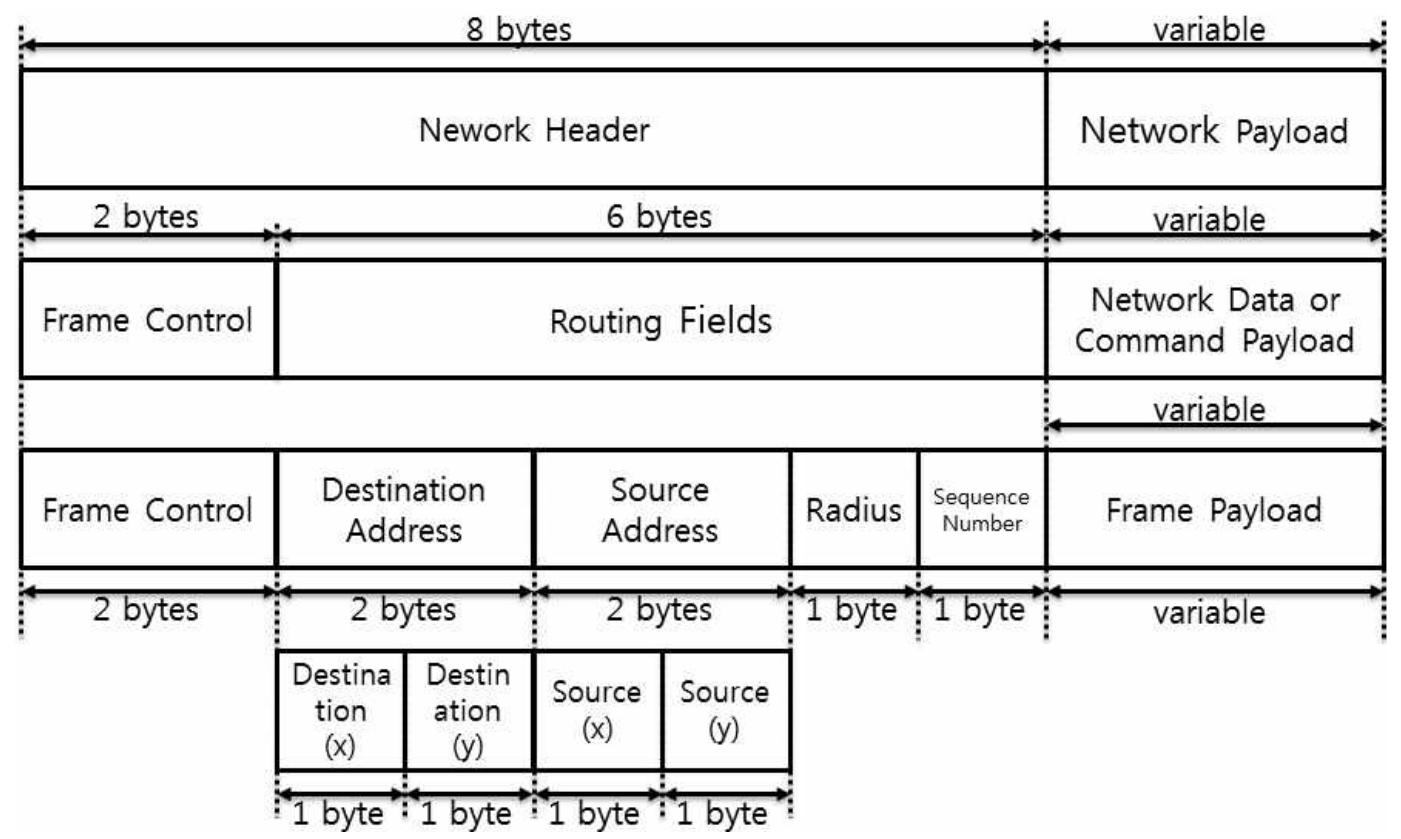

Fig. 2. Network layer frame format 
13]. As shown in Fig. 2, CBAAM improves the address space of the double-byte allocated to the destination and source addresses in the frame format of ZigBee network layer which can use the $x$ and $y$-axis of the node.

By utilizing two dimension coordinates, CBAAM assigns addresses to nodes. 8 -bit is allocated to $x$ and $y$-axis and these address field is used as the address of the node.

According to the research results, this method shows the number of hop count and operation speed is more efficient in comparison with the DAAM. Moreover, if the maximum communication distance of the node is assumed as $30 \mathrm{~m}$, the maximum connectable distance is suitable for the wide area network where the distance can reach to 7.6 $\mathrm{km}$.

Fig. 3 shows a building or apartment environment of HAN. In this case, ZigBee network devices are able to obtain their own ids by the combination of the $x, y$-coordinates. Parent nodes, such as a coordinator and root routers, connect with their child nodes directly or indirectly by their following $i d \mathrm{~s}$. This scheme assigns $x-y$ coordinates addresses ( 1 to 255,1 to 255 ) to nodes sequentially.

When direct connection of the node is impossible for a root router, a router can be additionally connected with the root router as a child node. In this case, the root router and routers know all the remaining addresses.

In order to assign addresses to the freshly added nodes, the root router and routers have to maintain the address table. In the worst case, the root router and routers should manage 255 nodes addresses, which are shown in Fig. 4.

\section{PROPOSED SCHEME}

In order to improve routing efficiency, transfer speed and network congestion, the proposed scheme modifies the coordinate-based address assignment method. Fig. 5 depicts our address as- signment scheme in detail, which can be applied to the building or apartment structure.

Different from the previous scheme, proposed method predefines the location of the coordinator and root routers in the intermediate area of the network. For example, the network address is assigned to the nodes from 0 to 255 , then $(127,127)$ indicates the coordinator, and $(x, 127)$ or $(127, y)$ indicates root routers. Address 127 can be expressed using the binary number 0111 1111. Based on this value, a big or small address is assigned to the child node by turns. In our scheme, thus, the number of hop count of the packet transmission is shortened than that of the previous method. In addition, the capacity of the routing table is also shortened.

As in Fig. 6, in order to assign addresses to freshly added nodes, the root router and routers have to maintain the address table. In the worst case, however, the root router and routers manage 127 nodes addresses and this address capacity is only 50\% compared with that of the existing scheme. We explain the differences with the CBAAM in below.

- Root router has the address of $(x, 127)$ or $(127, x)$. And a router connected to the root router is assigned the small or big number of address based on the address of the relevant root router.

- A node requesting the connection to a router is sequentially assigned address from 0 to 126 when the router address is smaller than 127. And address is bigger than 127, address is assigned from 128 to 254

The later procedure is identical with the sequential address assignment.

As shown in Fig. 6, node $N$ can be assigned address from 0 to 126 since the router address is less than 127. Thus, the address space assigned to the node is 0 from 115 . Therefore, the node is assigned 115.

The existing coordinate based address assignment scheme indicates that routing is possible 

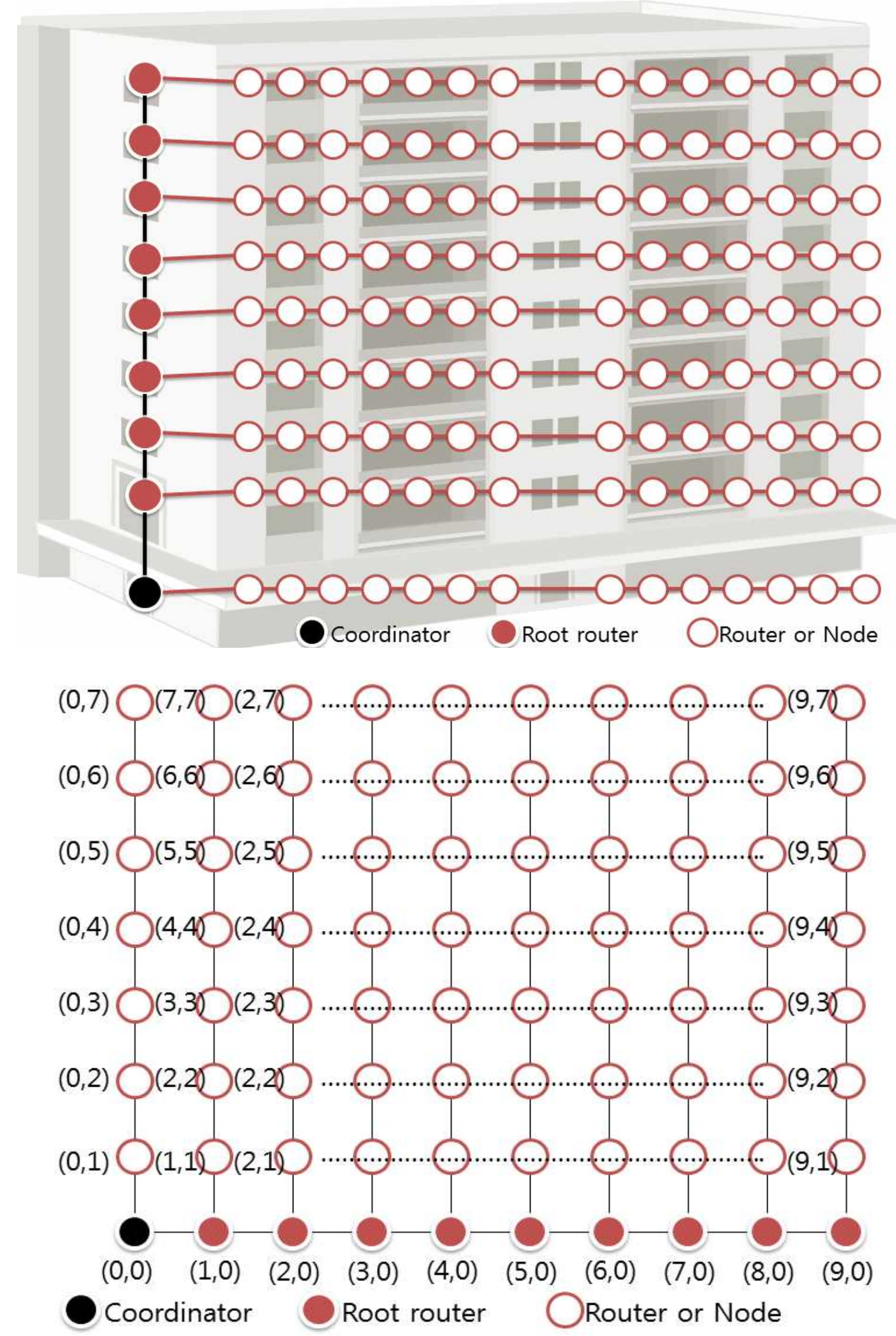

Fig. 3. Example of connected node in HAN and address assignment. 


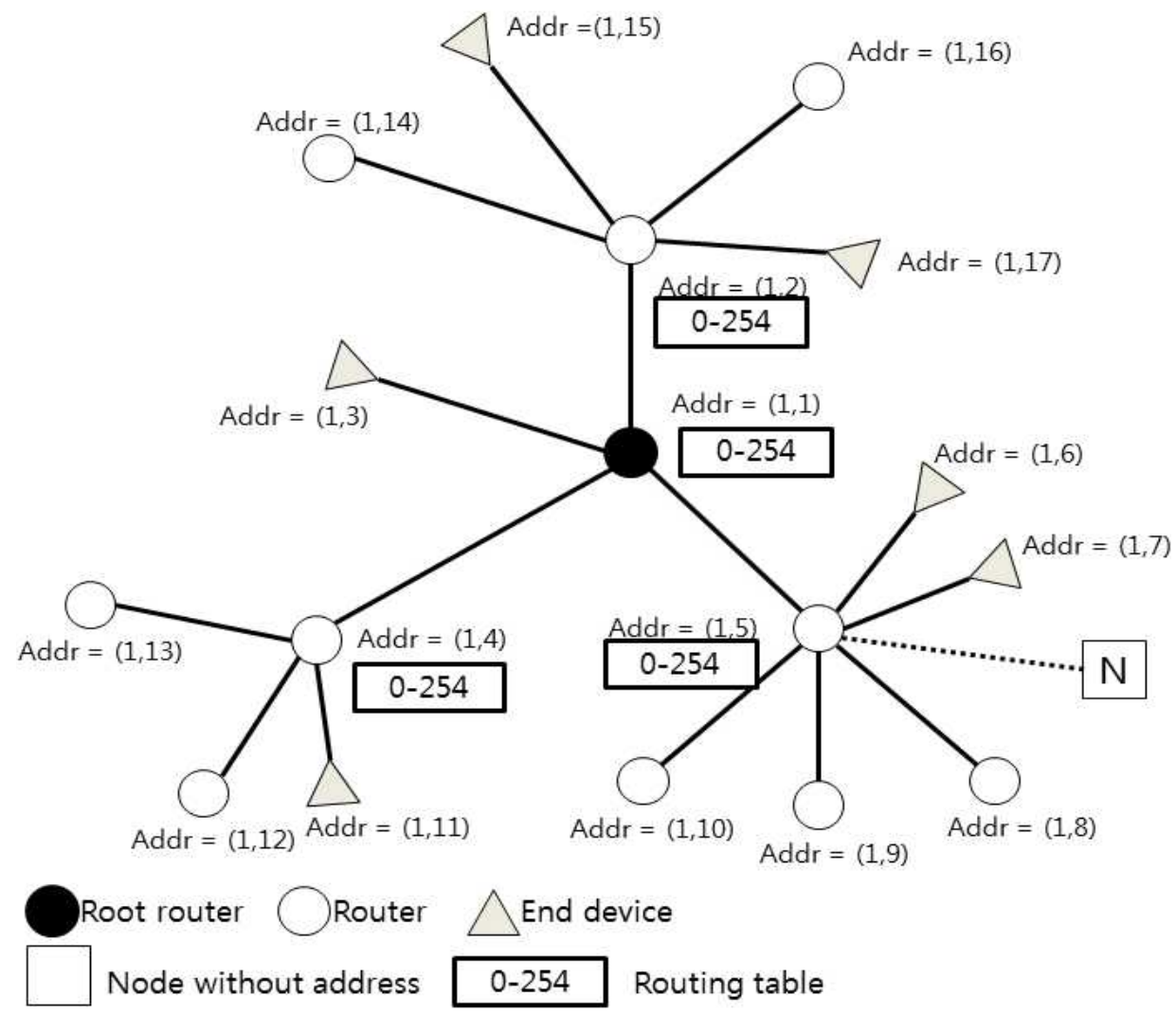

Fig. 4. Example of router and node address assignment.

through the comparison of $x$ or $y$ coordinates of the destination address. Thus, coordinate based scheme was 8 bits for address comparison, while the distribute address assignment scheme was 48 bits. As shown in Fig. 7, the proposed scheme is possible that route search through only 1 bit address comparison. It is a dramatic improvement.

Route search through the comparison of first 1 bit can be explained by the following example. Address 127 can be expressed using the binary number 0111 1111, and if the address is bigger than 128 , the binary expression is 10000000 . As depicted in Fig. 7, routers and the coordinator formation is a straight line which divide the area of the network into two parts. Thus, when the coordinates of source and destination node are in the different part of the network separately, the route search becomes possible with the comparison of first 1 bit about $50 \%$ of the path. Therefore, it is easily concluded that our scheme is more efficient. Fig. 7 shows that the example of coordinate comparison of the existing scheme and the proposed scheme.

\section{PERFORMANCE EVALUATION}

In this section, we present experimental results of address assignment scheme in terms of the average number of hop count, packet transfer time and network processing time by using MATLAB software. For fair evaluation, we evaluated the performance in the same manner of previous schemes. In this evaluation, we assumed the node transmission range is $50 \mathrm{~m}$.

\subsection{Comparison of average hop count}

In the mobile network, the number of hop count indicates that the routing cost. We measured the average hop count for our scheme to show the routing efficiency.

As shown in Fig. 8(a), the number of hop count 

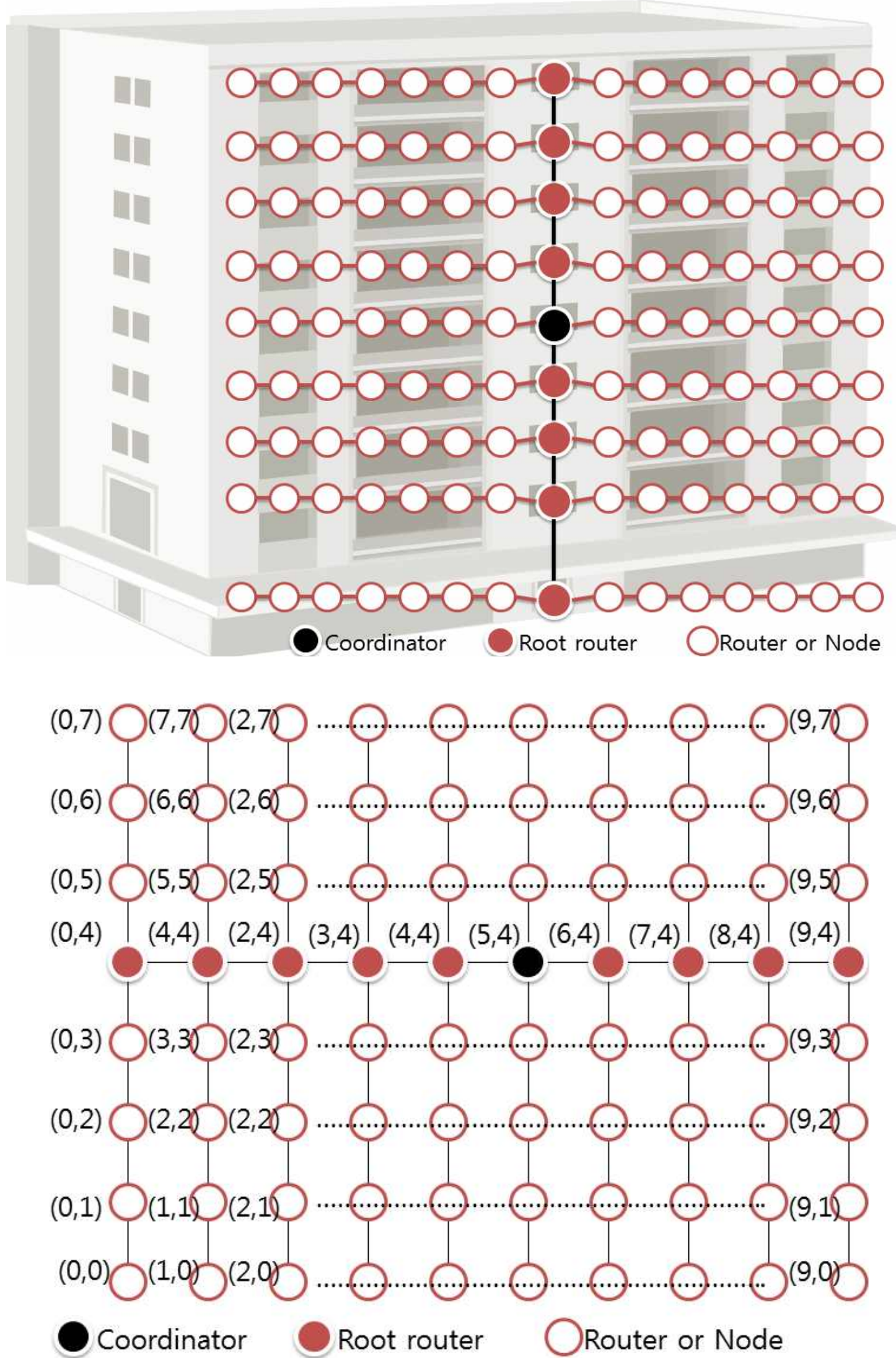

Fig. 5. Address assignment and applied example of the proposed scheme. 


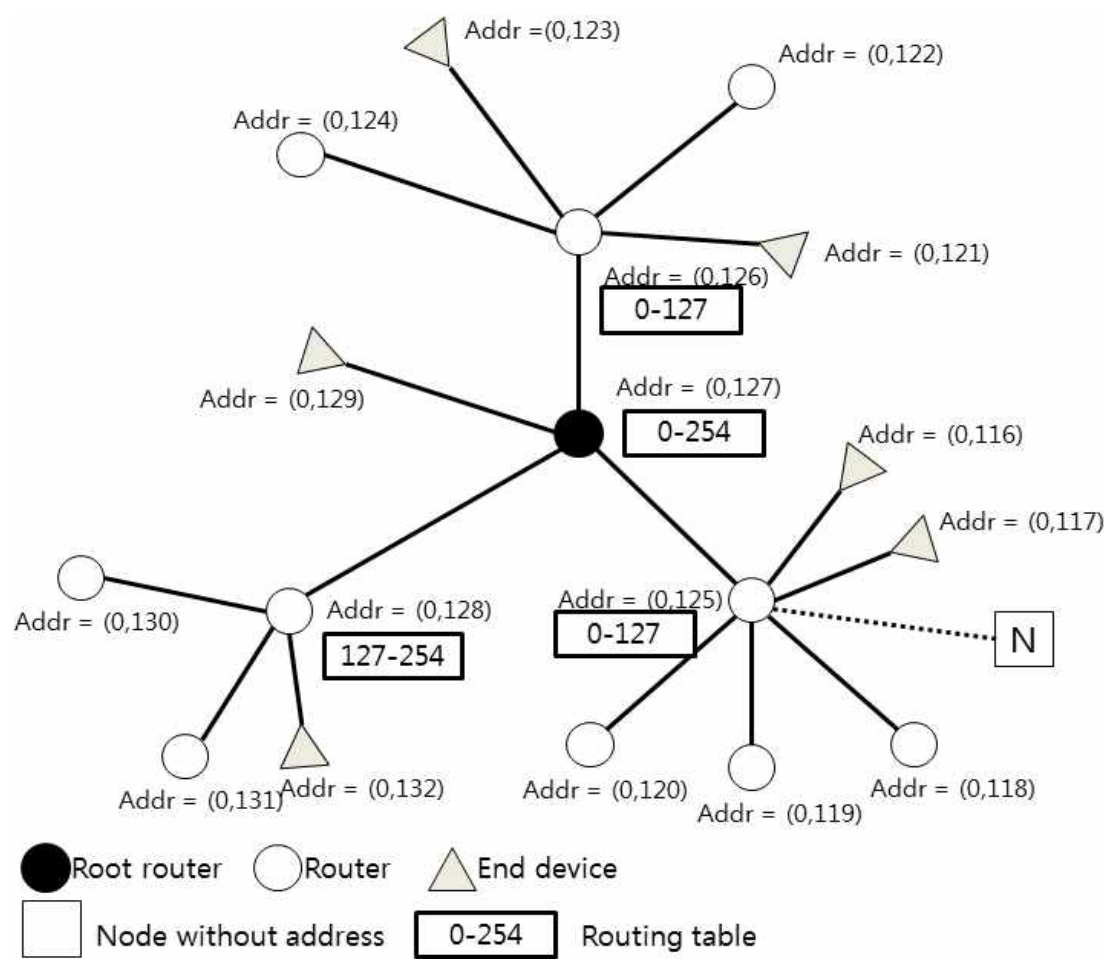

Fig. 6. Example of router and node address assignment.

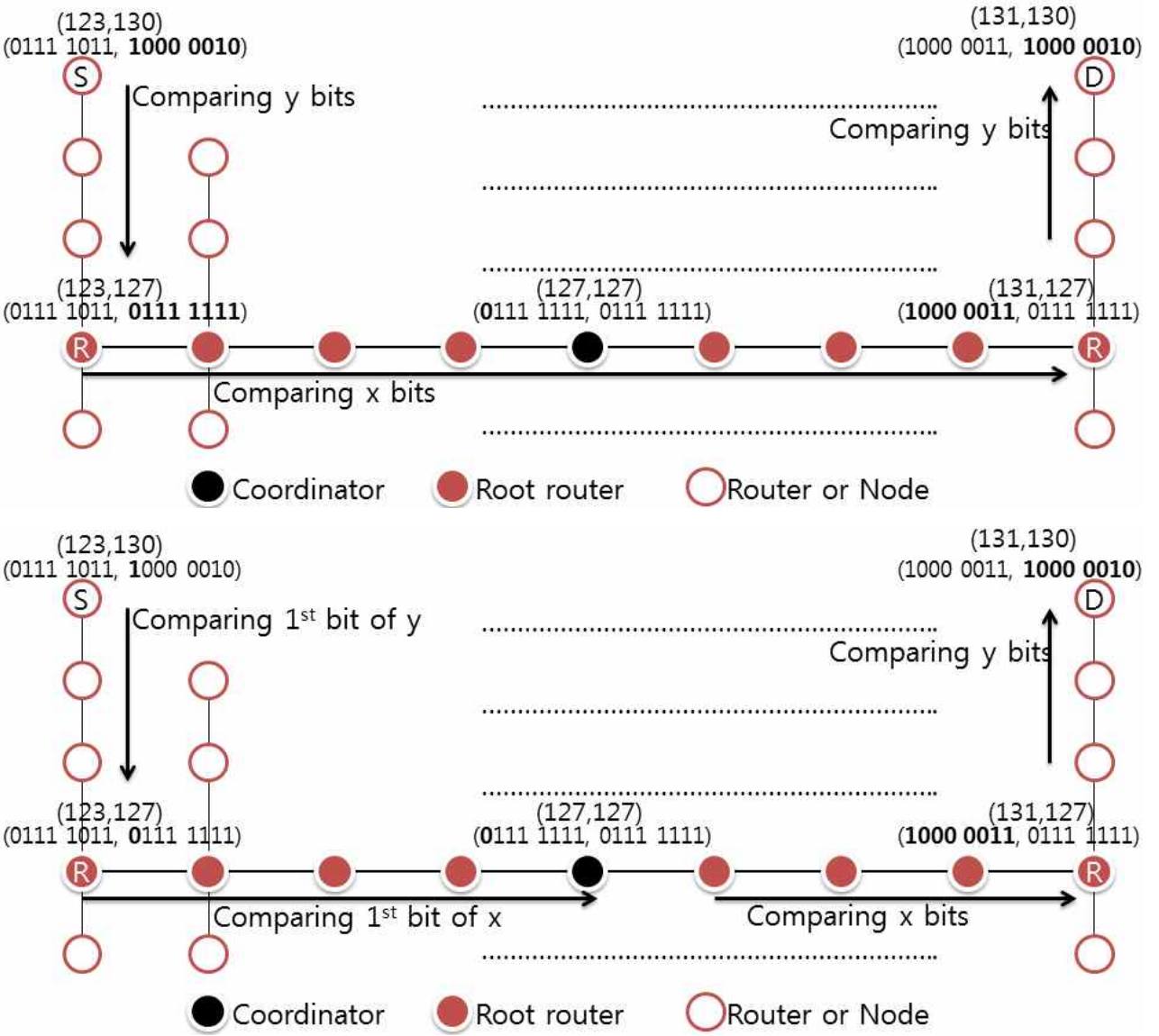

Fig. 7. Comparison of coordinates base and proposed scheme. 


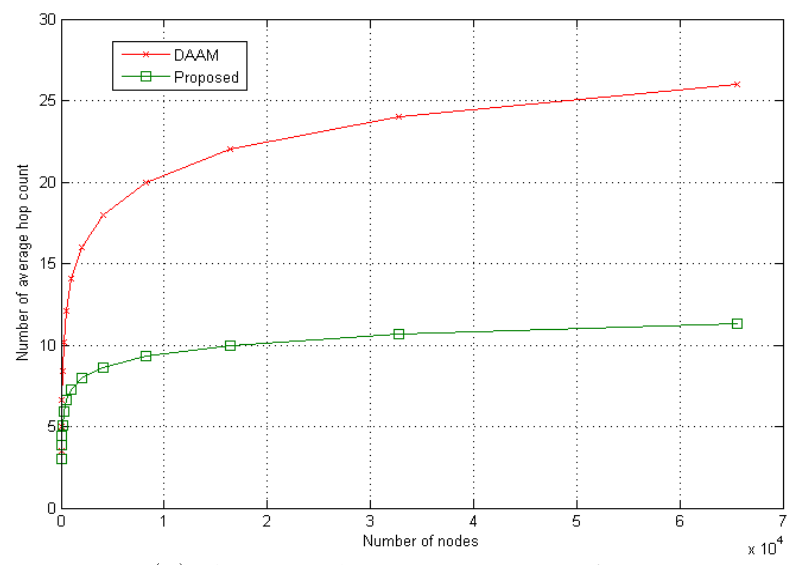

(a) Average hop count comparison

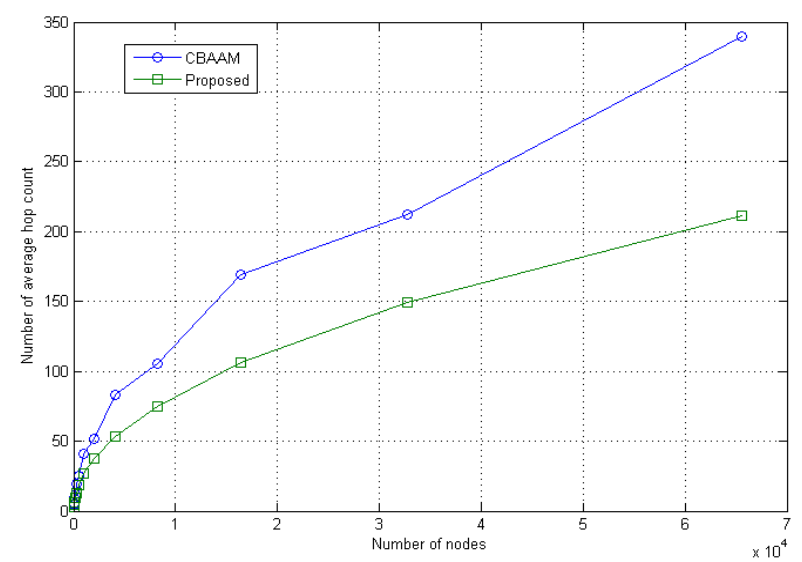

(b) Average hop count comparison in worst case of network connection

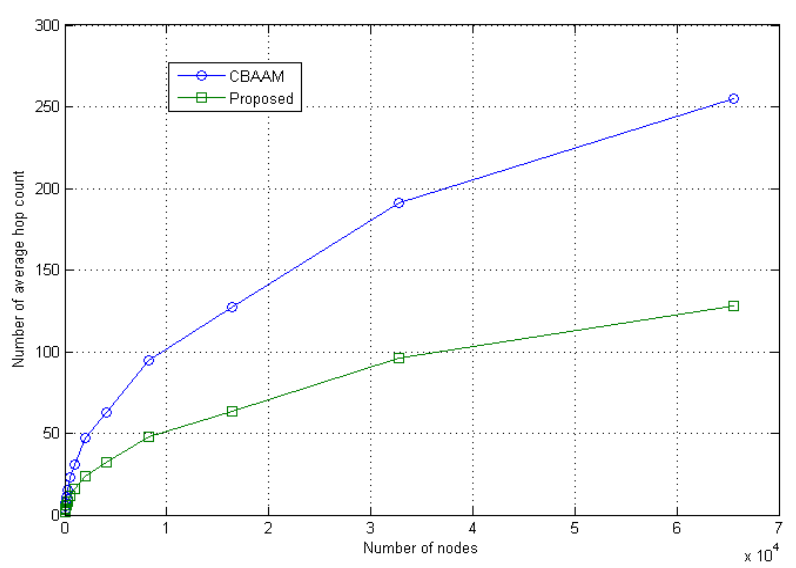

(c) Average hop count comparison from coordinator in worst case of network connection

Fig. 8. Comparison result of coordinates base and proposed scheme.

of the coordinate based schemes is shorter than that of DAAM. Because of using the same coordinate based address assignment scheme, the performance of CBAAM and our scheme are same.

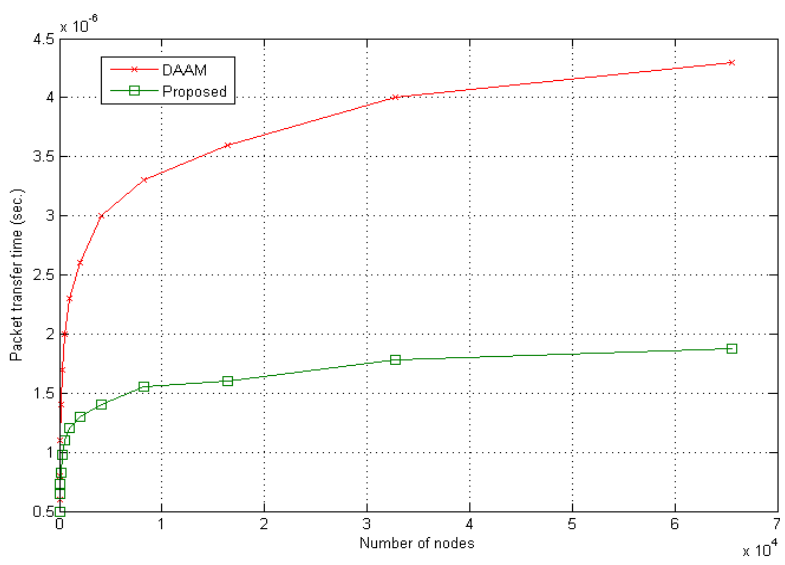

Fig. 9. Comparison result of packet transfer time.

In the case of all network devices are becoming routers, DAAM and CBAAM show unexpected result as shown in Fig. 8(b), and (c). However, our proposed scheme performs well.

\subsection{Comparison of packet transfer time}

As shown in Fig. 9, our scheme shows the same result of the CBAAM. However, when we consider the bit comparison, the result is changed.

\subsection{Network processing time comparison}

We compared the in-network packet processing time for the network performance evaluation. According to Fig. 10(a), the proposed scheme performs better than existing schemes. However, in the worst case of the network connection shown in Fig. 10(b), and (c), our scheme our scheme performs well. Due to the 1-bit comparison, in our scheme a router can easily find a path and then deliver the packet to the destination with low cost.

\section{CONCLUSION}

We propose a method to improve the coordinate base address assignment scheme. According to the experimental results, the network processing time is improved compared with the existing schemes. In addition, the size of address table maintained by a router reduced by $50 \%$. In order to determine the 


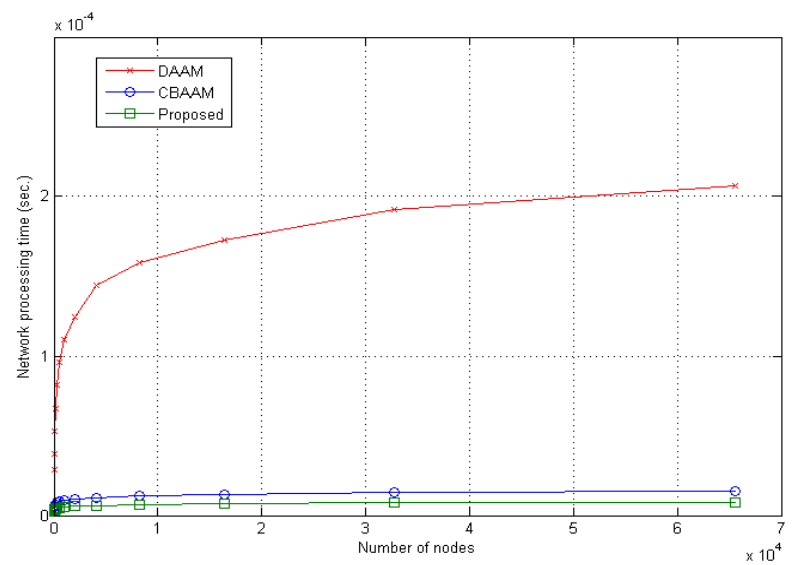

(a) Average network processing time comparison

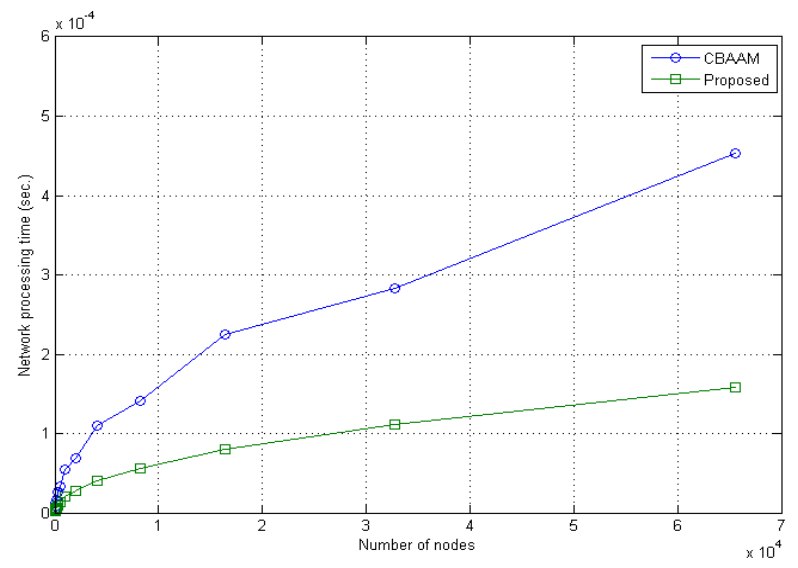

(b) Average network processing time comparison in worst case of network connection

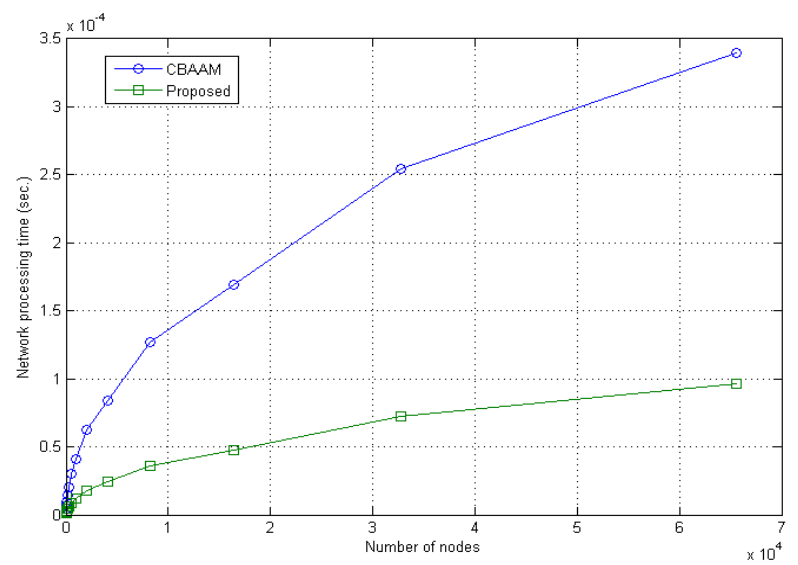

(c) Average network processing time comparison from coordinator in worst case of network connection

Fig. 10. Comparison result of network processing time.

route, a router refers to the routing table. This research shows that the route search of the bit unit can improve the network processing speed considerably.

\section{REFERENCES}

[1] U.S. Department of Commerce, NIST Framework and Roadmap for Smart Grid Interoperability Standards Release 1.0 (Draft), 2009.

[2] ZigBee Alliance, ZigBee Specification, ZigBee document 053474r17, 2008.

[3] Doohyun Kim and Sung Ho Cho," An Efficient Search Algorithm for Shorten Routing Path in ZigBee Networks," The Journal of Korea Information and Communication Society, Vol. 34, No. 12, pp. 1535-1541, 2009.

[4] Hyo Hyun Choi, "An Transmission Time Allocation Scheme in ZigBee Network," Journal of the Korea Society of Computer and Information, Vol. 16, No.4, pp. 121-128, 2011.

[5] Khusvinder Gill, Shuang-Hua Yang, Fang Yao, and Xin Lu, "A ZigBee-Based Home Automation System," IEEE Transactions on Consumer Electronics, Vol. 55, No. 2, pp. 422430, 2009.

[6] Jinsoo Han, Chang-Sic Choi, and Ilwoo Lee, "More Efficient Home Energy Management System Based on ZigBee Communication and Infrared Remote Controls," IEEE Transactions on Consumer Electronics, Vol. 57, No. 1, pp. 85-89, 2011.

[7] Seok-Cheol Lee, Shin Il Jeong, and ChangSoo Kim, "Design and Development of Monitoring System for Subway Station based on USN," Journal of Korea Multimedia Society, Vol. 12, No. 11, pp. 1629-1639, 2009.

[8] Meng-Shiuan Pan, Hua-Wei Fang, Yung-Chih Liu, and Yu-Chee Tseng, "Address Assignment and Routing Schemes for ZigBee-Based Long-Thin Wireless Sensor Networks," IEEE Vehicular Technology Conference, pp. 173177, 2008.

[9] Jongjun Park, Hoon Jeong, Soyoung Hwang, and Seong-Soon Joo, "A Hierarchical Cluster 
Tree Based Address Assignment Method for Large and Scalable Wireless Sensor Networks," The Journal of Korea Information and Communication Society, Vol. 34, No. 12, pp. 1514-1523, 2009.

[10] Fujun Ye and Ruifang Pan, “A Crosscut Tree Routing Scheme for Preemptive Distributed Address Assignment Mechanism," International Conference on Consumer Electronics, Communications and Networks, pp. 38083812, 2011.

[11] Zhi Ren, Pengxiang Li, and Jun Fang, "Segmentation-Based On-Demand Scalable Address Assignment for ZigBee Networks," IEEE Vehicular Technology Conference, pp. 1-5, 2011.

[12] Jaehyun Kim, Soojung Hur, Yongwan Park, Wonsek Kang, and Dongha Lee, "Effective address assignment method in hierarchical structure of Zigbee network," Journal of the Institute of Electronics Engineers of Korea, Vol. 44, No. 10, pp. 20-28, 2007.

[13] Yang-Hyun Cho, Song-Bin Lim, and GyungMok Kim, "An Efficient Addressing Scheme Using (x, y) Coordinates in Environments of Smart Grid," Journal of the Korea Society of Computer and Information, Vol. 17, No. 1, pp. 61-69, 2012.
[14] Dongmin Choi, Jian Shen, and Ilyong Chung, "A Study of ZigBee Address Assignment Scheme in Smart Grid Environment," Spring Conference of Korean Institute of Smart Media, pp. 57-59, 2012.

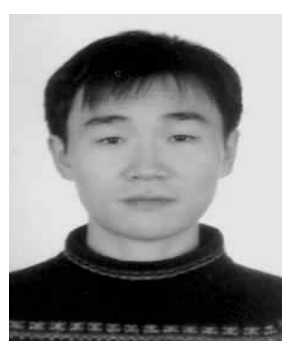

\section{Dongmin Choi}

He received his B.E. degree from the Kyunghee University in 2003 and M.S. and Ph.D. de ${ }^{-}$ grees in computer Science from Chosun University in 2007 and 2011, respectively. He is work ${ }^{-}$ ing as a researcher in Computer Science at Chosun University. His research interests are in information security, sensor network systems, mobile ad-hoc systems, smart grid home network systems and internet ethics.

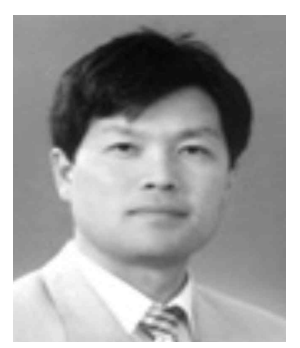

\section{Ilyong Chung}

He received the B.E. degree from Hanyang University, Seoul, Korea, in 1983 and the M.S. and Ph.D. degrees in Computer Science from City University of New York, in 1987 and 1991, respectively. From 1991 to 1994, he was a senior technical staff of Electronic and Telecommunication Research Institute (ETRI), Dajeon, Korea. Since 1994, he has been a Professor in Department of Computer Science, Gwangju, Korea. His research interests are in computer networking, security systems and coding theory. 